\title{
Directivity influence of signals propagation through EDFA gain medium of Brillouin- erbium fiber laser
}

\begin{abstract}
We experimentally demonstrate a multiwavelength Brillouinï erbium fiber laser in two configurations; uni-directional and bi-directional propagation of Brillouin pump and Brillouin Stokes signals through an Erbium-doped fiber gain. The influence of these configurations on the performance of the output parameters in terms of lasing threshold, output channel generation and tuning range of the generated output channels are investigated. We discovered that there is a trade-off between these two fiber laser configurations. The uni-directional amplifier configuration provides greater tuning range of $46.8 \mathrm{~nm}$ against $23 \mathrm{~nm}$ at maximum Brillouin pump power of $2 \mathrm{~mW}$ and $1480-\mathrm{nm}$ pump power of $130 \mathrm{~mW}$. On the other hand, the bi-directional amplifier configuration provides 13 output channels against 6 output channels obtained from the uni-directional amplifier configuration at the same pumping powers. Nevertheless, the bi-directional amplifier configuration requires much lower pump power to initiate lasing.
\end{abstract}

Keyword: Fibre lasers; Brillouin scattering; Multiwavelength 